\title{
Online social networking addiction and depression: The results from a large-scale prospective cohort study in Chinese adolescents
}

\author{
JI-BIN LI ${ }^{1,2 *}$, PHOENIX K. H. MO ${ }^{2,3}$, JOSEPH T. F. LAU ${ }^{2,3}$, XUE-FEN SU ${ }^{2,3}$, XI ZHANG ${ }^{4}$, ANISE M. S. WU ${ }^{5}$, \\ JIN-CHENG MAI ${ }^{6}$ and YU-XIA CHEN ${ }^{6}$
}

\author{
${ }^{1}$ Department of Clinical Research, State Key Laboratory of Oncology in South China, Collaborative Innovation Center for Cancer Medicine, \\ Sun Yat-sen University Cancer Center, Guangzhou, China \\ ${ }^{2}$ Centre for Health Behaviours Research, The Jockey Club School of Public Health and Primary Care, The Chinese University of Hong Kong, \\ Hong Kong, China \\ ${ }^{3}$ Shenzhen Research Institute, The Chinese University of Hong Kong, Shenzhen, China \\ ${ }^{4}$ Clinical Research Unit, Xin Hua Hospital - Shanghai Jiao Tong University School of Medicine, Shanghai, China \\ ${ }^{5}$ Faculty of Social Sciences, Department of Psychology, University of Macau, Macao, China \\ ${ }^{6}$ Department of Psychological Health Research, Center for Health Promotion of Primary and Secondary School of Guangzhou, \\ Guangzhou, China
}

(Received: April 19, 2018; revised manuscript received: July 16, 2018; accepted: July 28, 2018)

\begin{abstract}
Background and aims: The aim of this study is to estimate the longitudinal associations between online social networking addiction (OSNA) and depression, whether OSNA predicts development of depression, and reversely, whether depression predicts development of OSNA. Methods: A total of 5,365 students from nine secondary schools in Guangzhou, Southern China were surveyed at baseline in March 2014, and followed up 9 months later. Level of OSNA and depression were measured using the validated OSNA scale and CES-D, respectively. Multilevel logistic regression models were applied to estimate the longitudinal associations between OSNA and depression. Results: Adolescents who were depressed but free of OSNA at baseline had 1.48 times more likely to develop OSNA at follow-up compared with those non-depressed at baseline [adjusted OR (AOR): 1.48, 95\% confidence interval (CI): 1.14-1.93]. In addition, compared with those who were not depressed during the follow-up period, adolescents who were persistently depressed or emerging depressed during the follow-up period had increased risk of developing OSNA at follow-up (AOR: 3.45, 95\% CI: 2.51-4.75 for persistent depression; AOR: 4.47, 95\% CI: 3.33-5.99 for emerging depression). Reversely, among those without depression at baseline, adolescents who were classified as persistent OSNA or emerging OSNA had higher risk of developing depression compared with those who were no OSNA (AOR: 1.65, 95\% CI: 1.01-2.69 for persistent OSNA; AOR: 4.29; 95\% CI: 3.17-5.81 for emerging OSNA). Conclusion: The findings indicate a bidirectional association between OSNA and depression, meaning that addictive online social networking use is accompanied by increased level of depressive symptoms.
\end{abstract}

Keywords: online social networking addiction, depression, longitudinal association, adolescents

\section{INTRODUCTION}

Depression, the most widely reported psychiatric disorder (Knopf, Park, \& Mulye, 2008; Thapar, Collishaw, Potter, \& Thapar, 2010), is an important public health issue among adolescents. Over $9 \%$ of adolescents reported moderate to severe levels of depression, and its 1-year incidence rate was estimated at 3\% in the United States (Rushton, Forcier, \& Schectman, 2002). In Southern China, our previous study reported a 1 -week depression prevalence of $23.5 \%$ among secondary school students (Li et al., 2017).

A positive association between Internet addiction and depression among adolescents has been reported in both cross-sectional (Moreno, Jelenchick, \& Breland, 2015; Yoo, Cho, \& Cha, 2014) and longitudinal studies (Cho, Sung, Shin, Lim, \& Shin, 2013; Ko, Yen, Chen, Yeh, \& Yen,
2009; Lam, 2014). However, these studies assessed Internet addiction in general rather than specific types of online activities. Adolescents could conduct multiple types of online activities on the Internet. Several studies have highlighted the significance and necessity for distinguishing

\footnotetext{
* Corresponding authors: Ji-Bin Li, MD, PhD; Department of Clinical Research, State Key Laboratory of Oncology in South China, Collaborative Innovation Center for Cancer Medicine, Sun Yat-sen University Cancer Center, No. 651, Dong Feng East Road, Guangzhou 510060, China; Phone: +86 208734 3553; Fax: +86 208734 3535; E-mail: lijib@sysucc.org.cn; Joseph T. F. Lau, PhD, Professor; Centre for Health Behaviours Research, The Jockey Club School of Public Health and Primary Care, Prince of Wales Hospital, Shatin, Hong Kong, China; Phone: +852 2637 6606; Fax: +852 2645 3098; E-mail: jlau@cuhk.edu.hk
}

This is an open-access article distributed under the terms of the Creative Commons Attribution-NonCommercial 4.0 International License, which permits unrestricted use, distribution, and reproduction in any medium for non-commercial purposes, provided the original author and source are credited, a link to the CC License is provided, and changes - if any - are indicated. 
addiction to specific Internet-related activities from Internet addiction in general (Davis, 2001; Laconi, Tricard, \& Chabrol, 2015; Pontes, Szabo, \& Griffiths, 2015). Online social networking is a relatively new phenomenon, and high prevalence of depression has been observed among the population who are online social networking users (Lin et al., 2016; Tang \& Koh, 2017). Compared to the general population, teenagers and students are the most frequent users of online social networking (Griths, Kuss, \& Demetrovics, 2014). Online social networking addiction (OSNA) is a relatively new addictive behavior among adolescents along with compulsive involvement in online social networking activities. As a specific type of Internet-related behavioral addictions, OSNA incorporates core classic symptoms of addiction (Griffiths, 2013; Kuss \& Griffiths, 2011), and is defined as "being overly concerned about online social networking use, to be driven by a strong motivation to log on to or use online social networking that impairs other social activities, studies/jobs, interpersonal relationships, and/or psychological health and wellbeing" (Andreassen, 2015). OSNA has risen noticeably among adolescents. Around $9.78 \%$ of the US college students self-perceived to have Facebook addiction (Pempek, Yermolayeva, \& Calvert, 2009), and 29.5\% of Singaporean college students possess OSNA (Tang \& Koh, 2017). A study in 2010 reported that the OSNA prevalence was even higher than 30\% in Chinese college students (Zhou \& Leung, 2010). Evidences have suggested that excessive and compulsive online social networking is seldom beneficial, rather having potentially detrimental effects on adolescents' psychosocial well-being, including emotional, relational, and other healthrelated outcomes (Andreassen, 2015).

A few of cross-sectional surveys reported a positive association between OSNA and depression among adolescents (Hong, Huang, Lin, \& Chiu, 2014; Koc \& Gulyagci, 2013). However, due to the inherent limitation of the crosssectional study design, it is still unclear whether OSNA is a cause or consequence of depression or bidirectional. Online social networking could provide adolescents with social convenience and capital, selective self-disclosure, and potential social support (Ellison, Steinfield, \& Lampe, 2007; Steinfield, Ellison, \& Lampe, 2008). Individuals who experience psychiatric disorders (i.e., depression and anxiety) might view online social networking as a safe and important virtual community (Gámez-Guadix, 2014), where they could escape from emotional problems experienced in the real world (Andreassen, 2015; Griths et al., 2014), and further lead to potential addictive involvement (Oberst, Wegmann, Stodt, Brand, \& Chamarro, 2017). Meanwhile, excessive exposure to virtual community would result in negative emotions (McDougall et al., 2016). Adolescents with maladjustment to their depressive moods may experience more detrimental effects of excessive online social networking (Selfhout, Branje, Delsing, Ter Bogt, \& Meeus, 2009). Therefore, a bidirectional association between OSNA and depression is theoretically reasonable. However, to our knowledge, there is no prospective study that focused on exploring the longitudinal relationships between OSNA and depression among adolescents and other populations.

Therefore, we designed a prospective study to comprehensively estimate the longitudinal association between depression and OSNA over time, such as whether OSNA predicts development of depression, and whether depression predicts development of OSNA, by considering changes in OSNA and depression status (e.g., remission from disorder) during a 9-month follow-up period.

\section{METHODS}

\section{Study design}

This prospective cohort study was conducted in Guangzhou, Southern China. The baseline survey was conducted from March to April 2014, and the subsequent follow-up survey was conducted at a 9-month interval, using the same procedure.

\section{Participants and sampling}

Participants were recruited using a stratified cluster sampling method. One district/county was conveniently selected from each of three regions (i.e., core, suburb, and outer suburb regions) in Guangzhou, respectively (red dots in Figure 1). Three public secondary schools were then conveniently selected from each selected district/county, and a total of nine schools were thus selected. All the seventh- and the eighth-grade students within the selected schools were voluntarily invited to participate in the study. Anonymous questionnaire was self-administrated by participants in the classroom settings with the absence of any teacher, under the supervision of well-trained research assistants.

A total of 5,365 (response rate $=98.04 \%$ ) students completed the baseline survey. The two questionnaires of the same students were matched using last four digits of home telephone number, last four digits of parents' mobile phone number, last four digits of participants' identity card number, participants' date of birth, last letter of self and parents' spell name. Finally, 4,871 of 5,365 participants provided complete questionnaires at follow-up (follow-up rate $=$ $90.8 \%$ ). After excluding those who did not use online social networking $(n=643)$, a total of 4,237 participants were involved in our longitudinal study.

\section{Measures}

Depression. Level of depressive symptoms was measured using the 20-item Chinese version of the Center for Epidemiology Scale for Depression (CES-D). Its psychometric properties have been validated among Chinese adolescents (Chen, Yang, \& Li, 2009; Cheng, Yen, Ko, \& Yen, 2012; Lee et al., 2008; Wang et al., 2013). Higher scores indicate more severe level of depressive symptoms, with a total score ranging from 0 to 60 (Radloff, 1977). The Cronbach's $\alpha$ coefficients in this study were .86 at baseline and .87 at follow-up, showing a good internal reliability. Individual reporting a CES-D score $\geq 21$ is defined as a depressed case (Stockings et al., 2015). Following the previous studies (Penninx, Deeg, van Eijk, Beekman, \& Guralnik, 2000; Van Gool et al., 2003), change in depression status during follow-up period in this study was 


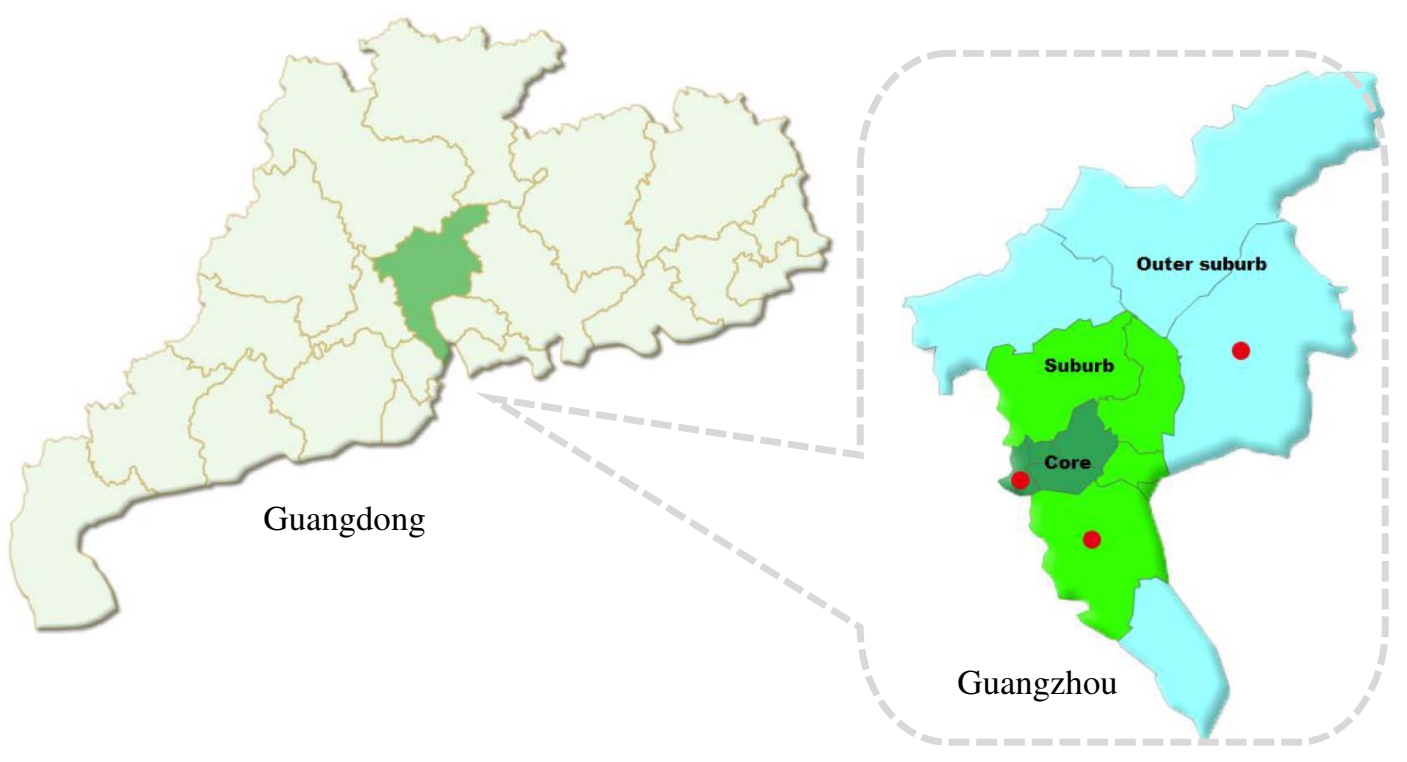

Figure 1. The location of the study sites

categorized as follows: no depression (participants without depression both at baseline and follow-up), remission from depression (participants with depression at baseline but transitioned to without depression at follow-up), persistent depression (participants with depression both at baseline and follow-up), and emerging depression (participants without depression at baseline but transitioned to with depression at follow-up).

Online social networking addiction (OSNA). Addictive level to online social networking was measured using an OSNA scale, which includes eight items measuring core addictive symptoms of cognitive and behavioral salience, conflict with other activities, euphoria, loss of control, withdrawal, relapse, and reinstatement. Higher scores of OSNA scale indicate higher levels of addictive tendency to online social networking, with a maximum score of 40 . Its psychometric properties have been thoroughly assessed in our previous study (Li et al., 2016). There is no established cut-off value for the OSNA scale to identify OSNA cases: participants who scored in the 10th decile of scores (i.e., OSNA score $\geq 24$ ) were classified as OSNA cases at baseline, and the same cut-off value was used to classify cases at follow-up. The similar classification strategy has been applied in the previous study (Verkuijl et al., 2014). The Cronbach's $\alpha$ coefficients of OSNA scale in this study were .86 at baseline and .89 at follow-up. Similarly, change in OSNA status from baseline to follow-up was categorized as follows: no OSNA (participants without OSNA both at baseline and follow-up), remission from OSNA (participants with OSNA at baseline but transitioned to without OSNA at follow-up), persistent OSNA (participants with OSNA both at baseline and follow-up), and emerging OSNA (participants without OSNA at baseline but transitioned to with OSNA at follow-up).

Covariates. Covariates included sex, grade, parental education levels, perceived family financial situation, living arrangement (with both parents or not), self-reported academic performance, and perceived study pressure at baseline.

\section{Statistical analyses}

Descriptive statistics (e.g., means, standard deviation, and percentages) were presented when appropriate. Intraclass correlation coefficients for clustering across schools were $1.56 \%(p=.002)$ for incident depression and $1.42 \%$ $(p=.042)$ for incident OSNA, indicating significant variances across schools (Wang, Xie, \& Fisher, 2009). Multilevel logistic regression models (Level 1: student; Level 2: school) were therefore applied to evaluate the longitudinal associations between OSNA and depression over time, accounting for the cluster sampling effect from school. Background covariates associated with incident depression/OSNA with $p<.05$ in univariate analysis or widely reported in the literature (i.e., sex and grade) were adjusted for in the multivariable logistic regression models.

For prediction of OSNA on new incidence of depression among participants who were non-depressed at baseline $(n=3,196)$, we first estimated the odds ratio (OR) of baseline OSNA, both binary variable (i.e., OSNA or not) and continuous variable (OSNA scale scores), on new incidence of depression after adjusting of significant covariates, and then further adjusting of baseline CES-D scale score (Hinkley et al., 2014). We then estimated the prediction of change in OSNA status over time on new incidence of depression, including a model adjusted of significant covariates and a model additionally adjusted of baseline CES-D scale score.

Reversely, the prediction of depression on new incidence of OSNA among participants without OSNA at baseline $(n=3,657)$ was estimated in a similar manner to that described above with new incidence of OSNA as outcome and depression as an exposure. The prediction of baseline depression (both continuous and categorical version) on new incidence of OSNA and prediction of change in depression status over time on new incidence of OSNA were estimated, respectively.

Statistical analyses were performed using SAS version 9.4 (SAS Institute, Cary, NC, USA). A two-sided $p$ value $<.05$ was considered statistically significant. 


\section{Ethics}

The study procedures were carried out in accordance with the Declaration of Helsinki. School consent and permission for the in-school survey were obtained from school principals before the survey had administered. Verbal consent was obtained from students before their participation. This study and the consent procedure were approved by the Survey and Behavioral Research Ethics Committee of the Chinese University of Hong Kong.

\section{RESULTS}

\section{Participants' characteristics and attrition analysis}

Attrition analysis showed that there were no significant differences in terms of parental education levels and selfreported academic performance between adolescents who were involved in the longitudinal analysis $(n=4,237)$ and who were excluded from longitudinal analysis $(n=1,128)$. Adolescents, who were involved in the longitudinal sample were more likely to be females, were from the eighth grade, have good family financial situation, lived with both parents, and perceive nil/light study pressure (Table 1).

Among 4,237 adolescents (mean age: 13.9, standard deviation: 0.7$)$ in the longitudinal sample, $49.7 \%$ (2,105 of $4,237)$ were female and $47.5 \%(2,011$ of 4,237$)$ were the seventh grade students. Most of adolescents $(88.4 \% ; 3,747$ of 4,237 ) were living with their parents. In the longitudinal sample, the prevalence of depression significantly increased from $24.6 \%(1,041$ of 4,237$)$ at baseline to $26.6 \%$ at followup (McNemar's test $=7.459, p=.006)$. There was no significant difference for the prevalence of OSNA between baseline and follow-up (13.7\% at baseline vs. $13.6 \%$ at follow-up; McNemar's test $=0.053, p=.818$ ). A total of 3,196 students were non-depressed at baseline, and 3,657 students were free of OSNA at baseline (Table 1).

\section{Potential confounders associated with new incidence of depression or OSNA}

Table 2 shows that perceived poor family financial situation, self-reported poor academic performance, and perceived heavy study pressure were significantly associated with both higher incidence of depression (range of univariate OR: 1.32-1.98) and higher incidence of OSNA (range of univariate OR: 1.61-2.76). Living with their parents was a significantly protective factor for incidence of OSNA only [univariate OR: $0.65,95 \%$ confidence interval (CI): $0.48-0.89]$.

\section{OSNA predict new incidence of depression}

Among 3,196 adolescents who were non-depressed at baseline, univariate model showed that baseline OSNA was significantly associated with higher incidence of depression during the follow-up period (univariate OR: $1.65,95 \% \mathrm{CI}$ : 1.22-2.22). After adjustment of sex, grade, family financial situation, academic performance, and perceived study pressure, the association remained significant [adjusted OR
(AOR): 1.48, 95\% CI: 1.09-2.01]. When further adjusting of baseline CES-D score, the association becomes statistically non-significant (AOR: 1.16, 95\% CI: 0.85-1.60). The similar results were observed when using OSNA score (continuous variable) as a predictor of new incident depression (Table 3).

We found a significant association between change in OSNA status and higher incidence of depression. Compared with adolescents who were classified as no OSNA, the risk of developing depression was 1.65 times (95\% CI: 1.01-2.69) higher among those with persistent OSNA, and 4.29 times (95\% CI: 3.17-5.81) higher among those with emerging OSNA, after adjustment of sex, grade, family financial situation, academic performance, perceived study pressure, and baseline CES-D scores (Table 3).

\section{Depression predict new incidence of OSNA}

Among 3,657 adolescents who were free of OSNA at baseline, univariate results demonstrated a significant positive association between baseline depression and higher incidence of OSNA (univariate OR: 2.02, 95\% CI: 1.58-2.58). After adjusting of sex, grade, family financial situation, living arrangement with parents, academic performance, and perceived study pressure, the association slightly attenuated but remained significant (AOR: 1.78, 95\% CI: 1.38-2.31). The association between baseline depression status and incidence of OSNA was still statistically significant when further adjustment of baseline OSNA scores (AOR: 1.48, 95\% CI: 1.14-1.93). The results were still significant when using CES-D score (continuous variable) as a predictor of new incident OSNA (Table 3).

A significant association between change in depression status and incidence of OSNA was observed in multivariable analysis. After adjusting of sex, grade, family financial situation, living arrangement with parents, academic performance, perceived study pressure, and baseline OSNA score, as compared to adolescents without depression, the odds of developing OSNA was 3.45 times (95\% CI: $2.51-4.75$ ) higher among those who were persistently depressed, and 4.47 times (95\% CI: 3.33-5.99) higher among those who were emerging depressed (Table 3 ).

\section{DISCUSSION}

In this large-scale longitudinal study, we found that adolescents who were depressed but free of ONSA at baseline had a $48 \%$ higher risk of developing OSNA within 9-month follow-up period compared with those without depression at baseline, but the prediction of baseline OSNA on new incidence of depression was not supported in this study. Moreover, when the effects of changes in status over time (i.e., remission from depression/OSNA at baseline to nondepression/non-OSNA at follow-up) were considered in the models, the results revealed a bidirectional association between OSNA and depression. Adolescents who were persistently depressed or emerging depressed had a higher risk of developing OSNA compared with those who were no depressed during the 9-month follow-up period. Reversely, adolescents who were persistent OSNA or emerging OSNA 
Li et al.

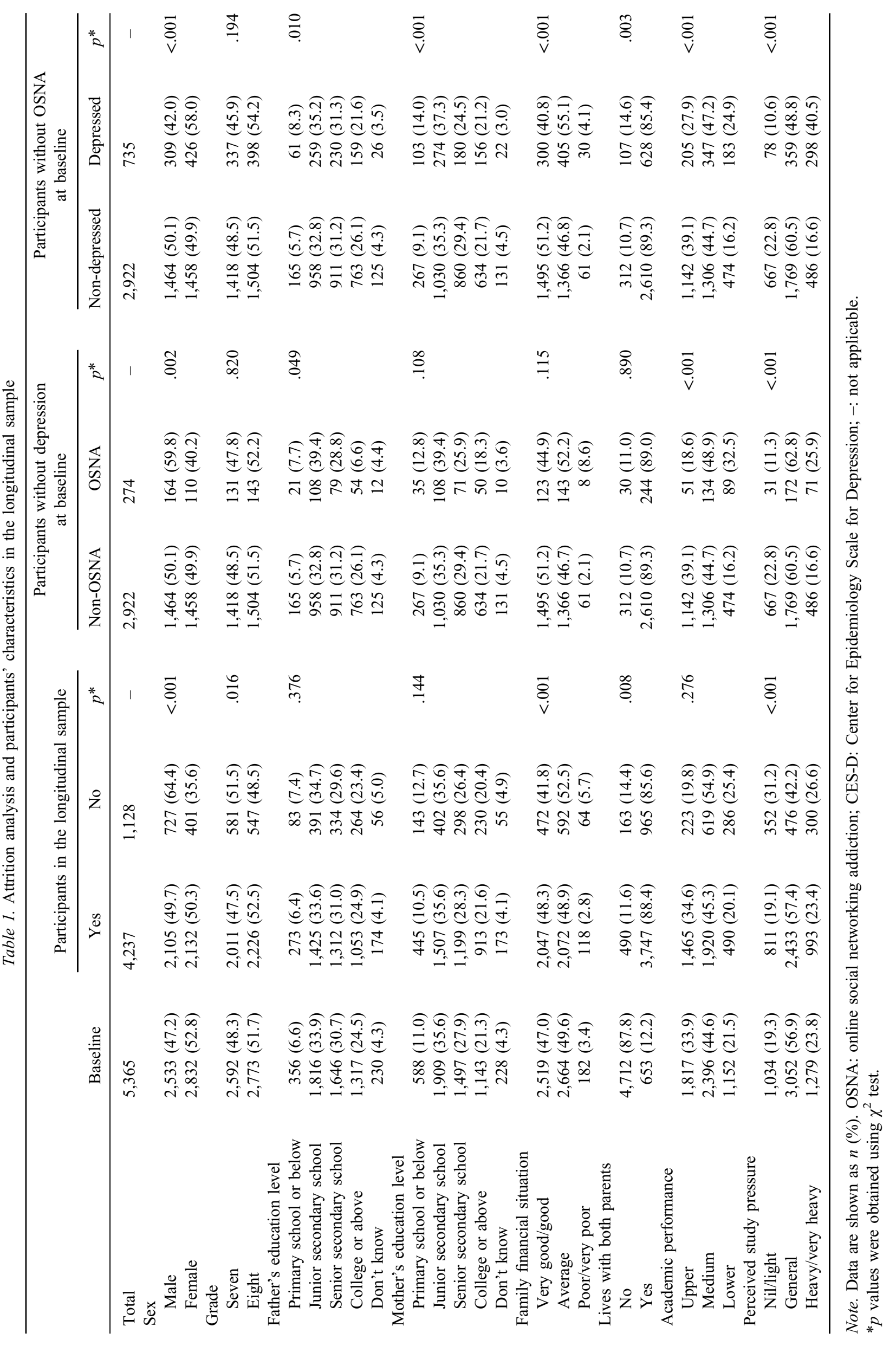


Table 2. Univariate associations between background covariates and incidence of depression/OSNA

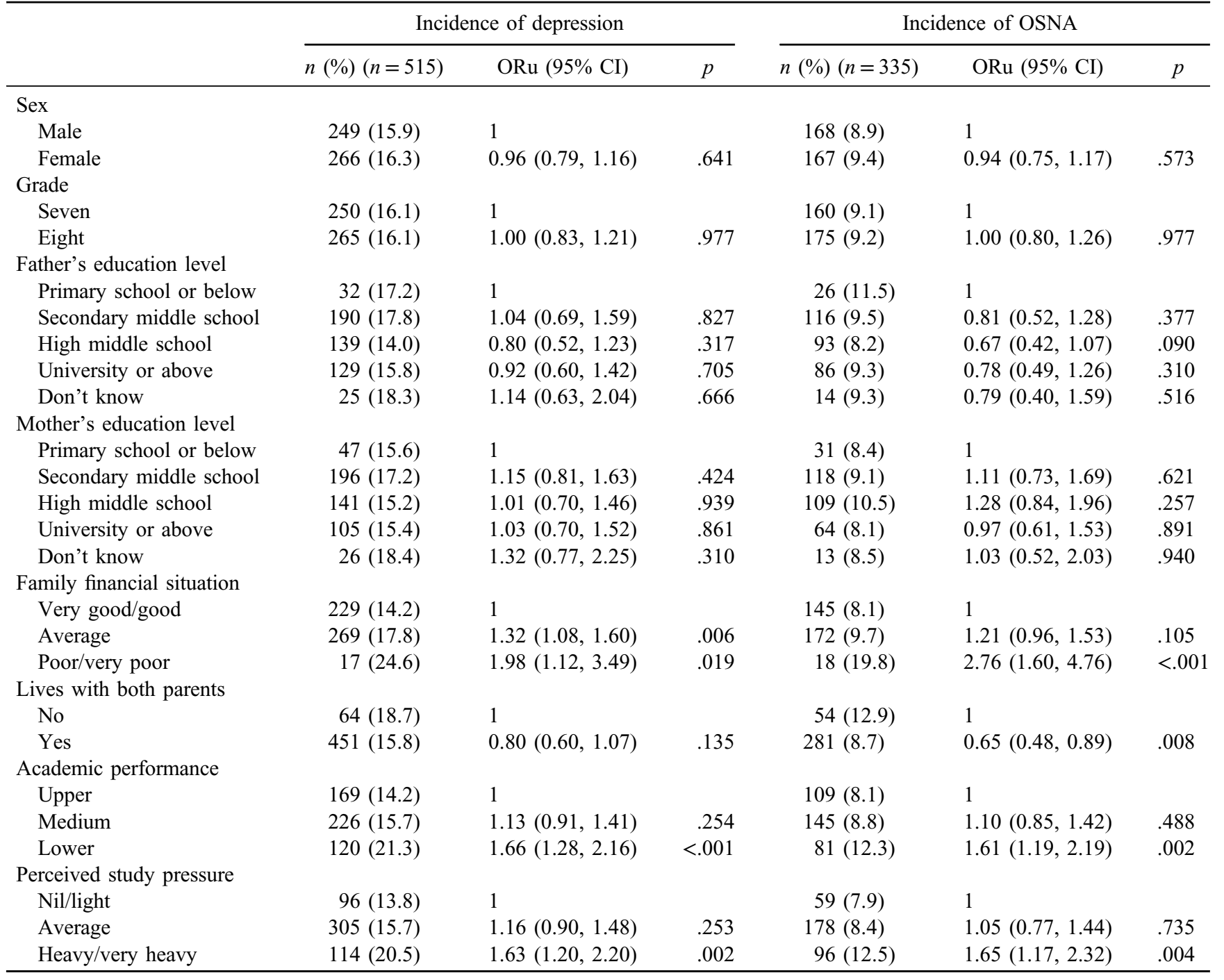

Note. OSNA: online social networking addiction; ORu: univariate odds ratio; $95 \% \mathrm{CI}$ : $95 \%$ confidence interval, obtained by the univariate logistic regression models.

also have an increased risk of developing depression compared with those who were no OSNA at both baseline and follow-up.

The difference in results obtained using baseline measures (i.e., baseline OSNA) and changes in status (i.e., change in OSNA status) to predict an incidence outcome (i.e., new incidence of depression) could be explained by the high remission rates from OSNA and depression during the follow-up period. The high natural remission rate of Internet addictive behaviors (49.5\%$51.5 \%$ ) has been observed in two previous longitudinal studies in Taiwan (Ko, Yen, Yen, Lin, \& Yang, 2007; Ko et al., 2015). The results from our previous survey in Hong Kong also consistently observed a high incidence of remission from Internet addiction behavior during a 12-month period (59.29 per 100 person-years; Lau, Wu, Gross, Cheng, $\&$ Lau, 2017). Similarly, in this study, a large proportion of remission cases from depression $(41.4 \%)$ and OSNA $(58.8 \%)$ were observed during the study period. These results indicated that OSNA and depression status in baseline assessment could not be treated as unchangeable conditions over time and hence ignoring the remission effect over time would potentially underestimate the effect of OSNA on depression. Thus, we speculated that the modeling approach involving dynamic changes in OSNA and depression status over time could provide more convincing and robust estimation by ruling out the potential offset effects from remission cases.

The findings in this study suggest a bidirectional association between OSNA and depression among adolescents, indicating that depression renders an individual vulnerability to develop OSNA, and in turn, the negative consequence of OSNA further exacerbates the symptoms of depression. Maladaptive cognitions (i.e., rumination, self-doubt, low self-efficacy, and negative self-appraisal) and dysfunctional behaviors (i.e., using Internet to escape from emotional problems) are critical in the development of Internet-related addictive behaviors (Davis, 2001). Depressed individuals usually present cognitive symptoms and possess positive expectancies for their Internet use that Internet could distract 
Li et al.

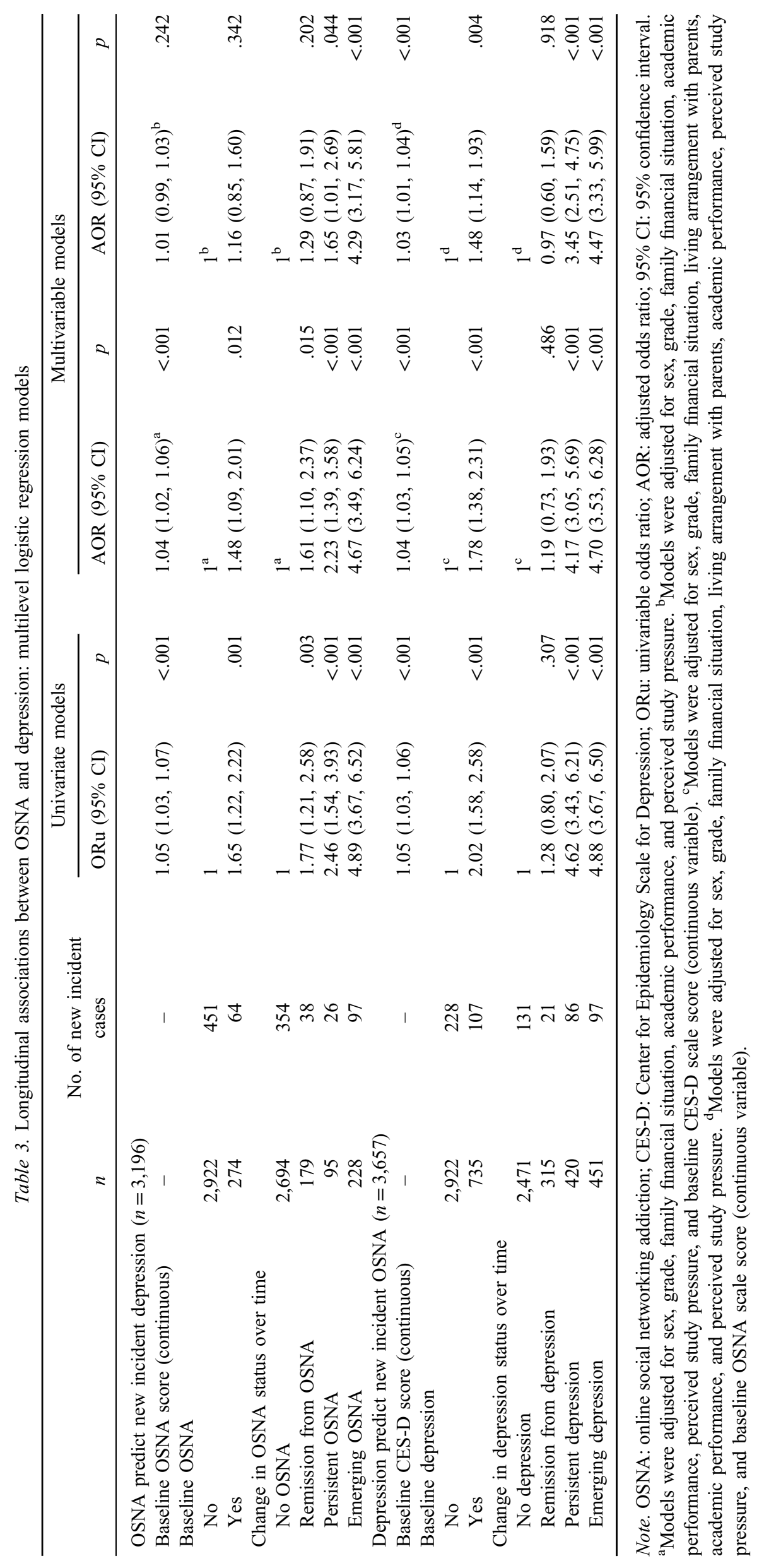


them from negative moods and personal problems (e.g., depression and loneliness; Brand, Laier, \& Young, 2014; Wu, Cheung, Ku, \& Hung, 2013). In particular, online social networking is attractive to people with mood problems because of its anonymity and absence of social cues (i.e., facial expression, voice inflection, and eye contact) compared to face-to-face communications (Young \& Rogers, 1998). Depressed individuals might prefer online social networking as a more secure and less threatening means of communication, as well as a means for regulating their negative moods (i.e., alleviating negative emotions, anxiety, and personal problems). These maladaptive cognition and avoidance coping strategies accelerate the development of OSNA. Excessive online social networking involvement displaces the time spent with family and peers in the real world, and causes withdrawal from interpersonal offline activities, which intensifies the negative moods (e.g., depressive symptoms and loneliness; Kraut et al., 1998), thereby presenting a reciprocal relation.

The findings in this study entail several implications in designing prevention and intervention programs. First, the positive prediction of baseline depression on new incidence of OSNA implies that depressed adolescents are at high risk of developing OSNA later. Intervention strategies of reducing depressive symptoms, that is, reducing maladaptive belief of positive outcome expectancies of Internet use, training social skills, and planning offline leisure activities (Chou et al., 2015), might effectively prevent the development of OSNA. Second, it is meaningful to assess the levels of depressive symptoms as a marker of the vulnerability for OSNA. Interventions and preventions targeting adolescents at high risk with identified depressive symptoms might reduce the odds of experiencing OSNA among school adolescents. Third, for the strong prediction of change in OSNA status (i.e., persistent OSNA and emerging OSNA) on incidence of depression and the prediction of change in depression status (i.e., persistent depression and emerging depression) on incidence of OSNA, it implies that OSNA is highly comorbid with depression, indicating a negative reinforcement mechanism.

There are some implications for future research. First, our results along with previous studies indicated that the level of OSNA and depressive symptoms are dynamic and reversible during the study period rather than random fluctuation in chance (Lau et al., 2017). Future studies involving measures of depression or OSNA are suggested to measure these disorders repeatedly rather than just one time point by assuming them unchangeable over time. In addition, the statistical methodology should consider such status change in modeling specifications, such as using change in pathological status over time rather than baseline status as a predictor of mental health outcomes. Second, it raised a concern whether these disorders (i.e., depressive symptoms and Internet-related behaviors) are long-lasting or shortterm. Further longitudinal studies involving latent-class trajectory modeling approach are alternative to estimate the natural developmental course of these disorders.

To our knowledge, our cohort study is the first to estimate a bidirectional association between OSNA and depression among the adolescents. The main strength of this study is a prospective large-scale study design with repeated measures for OSNA and depression. Another major advantage is that a bidirectional association, including the longitudinal prediction of OSNA on development of depression and the longitudinal prediction of depression on development of OSNA, was tested in the same sample.

However, several limitations should be noted when interpreting the findings. First, due to self-reported data collection method, reporting bias may consequentially exist (e.g., social desirable bias and recall bias). Second, this study focused on specific demographic population (i.e., nonclinical, school-based students), and the generalizability of the results to other population should be cautious. Studies in other demographic population (i.e., psychiatric clinical population) are necessary to further confirm such longitudinal associations found in this study. Third, there may exist misclassification for depression as a source of measurement error considering that depression was measured by a selfadministered epidemiological screening scale rather than clinical diagnosis to assess depression. Fourth, this study was restricted to two time points with 9-month interval. As we defined change in OSNA/depression (i.e., persistent ONSA/depression and remission from OSNA/depression) by comparing results of baseline and follow-up surveys that were conducted 9 months apart, we do not know whether OSNA/depression status changed or fluctuated during the 9-month period. Longitudinal studies with multiple observations and short time interval are necessary to capture the dynamic picture of these negative conditions. Fifth, considering that there is no available golden standard instrument and diagnostic criteria for OSNA, we used 10th decile of the OSNA scores at baseline to define OSNA cases following similar published study (Verkuijl et al., 2014). The sensitivity and specificity of such criterion for OSNA status is unclear and need to be evaluated in future research. However, the OSNA scale showed acceptable psychometric properties in this study and our previous studies. Sixth, the longitudinal associations between OSNA and depression were estimated separately using two subsamples. We believe that using pathological status as outcome rather than continuous scores could provide more meaningful explanation in epidemiological study. Cross-lagged structural equation modeling could be an alternative approach to explore causal directions in future longitudinal studies with three or more observations. In addition, our findings provide strong evidences of temporal associations (one important criterion for causal inference) between OSNA and depression. However, we could not rule out the possibility that a third variable not included in this study linked the longitudinal associations between OSNA and depression.

\section{CONCLUSIONS}

This study revealed a bidirectional association between OSNA and depression among adolescents, meaning that depression significantly contributes to the development of OSNA, and in turn, depressed individuals experience more deleterious effects from addictive online social networking use. More longitudinal studies with multiple observational time points and short-time interval are warranted for further confirmation of the findings from this study. 
Funding sources: The study was supported by National Science Foundation of China (no.: 81373021), and by the Jockey Club School of Public Health and Primary Care Research Postgraduate Students' Research Grants and CUHK Research Postgraduate Student Grants for Overseas Academic Activities in the Chinese University of Hong Kong. The funding sources had no role in the design and conduct of the study collection, management, analysis, and interpretation of the data; preparation, review, or approval of the manuscript; and decision to submit the manuscript for publication.

Authors' contribution: J-BL, JTFL, PKHM, and X-FS conceived and designed the study. J-BL, J-CM, and Y-XC acquired the data. J-BL, JTFL, and PKHM performed the statistical analyses. J-BL, JTFL, PKHM, XZ, and AMSW drafted and revised the manuscript. All authors contributed to the interpretation of the results and critical revision of the manuscript for important intellectual content and approved the final version of the manuscript.

Conflict of interest: The authors declare no conflict of interest.

Acknowledgements: The authors would like to appreciate all participants and their families and schools for supporting this study.

\section{REFERENCES}

Andreassen, C. S. (2015). Online social network site addiction: A comprehensive review. Current Addiction Reports, 2(2), 175-184. doi:10.1007/s40429-015-0056-9

Brand, M., Laier, C., \& Young, K. S. (2014). Internet addiction: Coping styles, expectancies, and treatment implications. Frontiers in Psychology, 5, 1256. doi:10.3389/fpsyg.2014.01256

Chen, Z. Y., Yang, X. D., \& Li, X. Y. (2009). Psychometric features of CES-D in Chinese adolescents. Chinese Journal of Clinical Psychology, 17(4), 443-448. doi:10.16128/j.cnki. 1005-3611.2009.04.027

Cheng, C. P., Yen, C. F., Ko, C. H., \& Yen, J. Y. (2012). Factor structure of the Center for Epidemiologic Studies Depression Scale in Taiwanese adolescents. Comprehensive Psychiatry, 53(3), 299-307. doi:10.1016/j.comppsych.2011.04.056

Cho, S. M., Sung, M. J., Shin, K. M., Lim, K. Y., \& Shin, Y. M. (2013). Does psychopathology in childhood predict Internet addiction in male adolescents? Child Psychiatry \& Human Development, 44(4), 549-555. doi:10.1007/s10578-012-0348-4

Chou, W. P., Ko, C. H., Kaufman, E. A., Crowell, S. E., Hsiao, R. C., Wang, P. W., Lin, J. J., \& Yen, C. F. (2015). Association of stress coping strategies with Internet addiction in college students: The moderating effect of depression. Comprehensive Psychiatry, 62, 27-33. doi:10.1016/j.comppsych.2015.06.004

Davis, R. A. (2001). A cognitive-behavioral model of pathological Internet use. Computers in Human Behavior, 17(2), 187-195. doi:10.1016/S0747-5632(00)00041-8

Ellison, N. B., Steinfield, C., \& Lampe, C. (2007). The benefits of Facebook "friends:" Social capital and college students' use of online social network sites. Journal of Computer-Mediated Communication, 12(4), 1143-1168. doi:10.1111/j.1083-6101. 2007.00367.x

Gámez-Guadix, M. (2014). Depressive symptoms and problematic Internet use among adolescents: Analysis of the longitudinal relationships from the cognitive-behavioral model. Cyberpsychology, Behavior, and Social Networking, 17(11), 714-719. doi:10.1089/cyber.2014.0226

Griffiths, M. D. (2013). Social networking addiction: Emerging themes and issues. Journal of Addiction Research \& Therapy, 4(5), e118. doi:10.4172/2155-6105.1000e118

Griths, M. D., Kuss, D. J., \& Demetrovics, Z. (2014). Social networking addiction: An overview of preliminary findings. In K. P. Rosenberg \& L. C. Feder (Eds.), Behavioral addictions: Criteria, evidence and treatment (pp. 119-141). London, UK: Elsevier.

Hinkley, T., Verbestel, V., Ahrens, W., Lissner, L., Molnár, D., Moreno, L. A., Pigeot, I., Pohlabeln, H., Reisch, L. A., \& Russo, P. (2014). Early childhood electronic media use as a predictor of poorer well-being: A prospective cohort study. JAMA Pediatrics, 168(5), 485-492. doi:10.1001/jamapediatrics.2014.94

Hong, F. Y., Huang, D. H., Lin, H. Y., \& Chiu, S. L. (2014). Analysis of the psychological traits, Facebook usage, and Facebook addiction model of Taiwanese university students. Telematics and Informatics, 31(4), 597-606. doi:10.1016/j. tele.2014.01.001

Knopf, D., Park, M. J., \& Mulye, T. P. (2008). The mental health of adolescents: A national profile, 2008. San Francisco, CA: National Adolescent Health Information Center.

Ko, C. H., Wang, P. W., Liu, T. L., Yen, C. F., Chen, C. S., \& Yen, J. Y. (2015). Bidirectional associations between family factors and Internet addiction among adolescents in a prospective investigation. Psychiatry and Clinical Neurosciences, 69(4), 192-200. doi:10.1111/pcn.12204

Ko, C. H., Yen, J. Y., Chen, C. S., Yeh, Y. C., \& Yen, C. F. (2009). Predictive values of psychiatric symptoms for Internet addiction in adolescents: A 2-year prospective study. Archives of Pediatrics \& Adolescent Medicine, 163(10), 937-943. doi:10.1001/archpediatrics.2009.159

Ko, C. H., Yen, J. Y., Yen, C. F., Lin, H. C., \& Yang, M. J. (2007). Factors predictive for incidence and remission of Internet addiction in young adolescents: A prospective study. CyberPsychology \& Behavior, 10(4), 545-551. doi:10.1089/cpb. 2007.9992

Koc, M., \& Gulyagci, S. (2013). Facebook addiction among Turkish college students: The role of psychological health, demographic, and usage characteristics. Cyberpsychology, Behavior, and Social Networking, 16(4), 279-284. doi:10.1089/ cyber.2012.0249

Kraut, R., Patterson, M., Lundmark, V., Kiesler, S., Mukopadhyay, T., \& Scherlis, W. (1998). Internet paradox. A social technology that reduces social involvement and psychological wellbeing? American Psychologist, 53(9), 1017-1031. doi:10. 1037/0003-066X.53.9.1017

Kuss, D. J., \& Griffiths, M. D. (2011). Online social networking and addiction - A review of the psychological literature. International Journal of Environmental Research and Public Health, 8(9), 3528-3552. doi:10.3390/ijerph8093528

Laconi, S., Tricard, N., \& Chabrol, H. (2015). Differences between specific and generalized problematic Internet uses according to gender, age, time spent online and psychopathological 
symptoms. Computers in Human Behavior, 48, 236-244. doi:10.1016/j.chb.2015.02.006

Lam, L. T. (2014). Internet gaming addiction, problematic use of the Internet, and sleep problems: A systematic review. Current Psychiatry Reports, 16(4), 444. doi:10.1007/s11920-0140444-1

Lau, J. T. F., Wu, A. M. S., Gross, D. L., Cheng, K. M., \& Lau, M. M. C. (2017). Is Internet addiction transitory or persistent? Incidence and prospective predictors of remission of Internet addiction among Chinese secondary school students. Addictive Behaviors, 74, 55-62. doi:10.1016/j.addbeh.2017.05.034

Lee, S. W., Stewart, S. M., Byrne, B. M., Wong, J. P. S., Ho, S. Y., Lee, P. W. H., \& Lam, T. H. (2008). Factor structure of the Center for Epidemiological Studies Depression Scale in Hong Kong adolescents. Journal of Personality Assessment, 90(2), 175-184. doi:10.1080/00223890701845385

Li, J. B., Lau, J. T. F., Mo, P. K. H., Su, X. F., Tang, J., Qin, Z. G., $\&$ Gross, D. L. (2017). Insomnia partially mediated the association between problematic Internet use and depression among secondary school students in China. Journal of Behavioral Addictions, 6(4), 554-563. doi:10.1556/2006.6.2017.085

Li, J. B., Lau, J. T. F., Mo, P. K. H., Su, X. F., Wu, A. M., Tang, J., \& Qin, Z. G. (2016). Validation of the Social Networking Activity Intensity Scale among junior middle school students in China. PLoS One, 11(10), e0165695. doi:10.1371/journal. pone. 0165695

Lin, L. Y., Sidani, J. E., Shensa, A., Radovic, A., Miller, E., Colditz, J. B., Hoffman, B. L., Giles, L. M., \& Primack, B. A. (2016). Association between social media use and depression among U. S. young adults. Depression and Anxiety, 33(4), 323-331. doi:10.1002/da.22466

McDougall, M. A., Walsh, M., Wattier, K., Knigge, R., Miller, L., Stevermer, M., \& Fogas, B. S. (2016). The effect of social networking sites on the relationship between perceived social support and depression. Psychiatry Research, 246, 223-229. doi:10.1016/j.psychres.2016.09.018

Moreno, M. A., Jelenchick, L. A., \& Breland, D. J. (2015). Exploring depression and problematic Internet use among college females: A multisite study. Computers in Human Behavior, 49, 601-607. doi:10.1016/j.chb.2015.03.033

Oberst, U., Wegmann, E., Stodt, B., Brand, M., \& Chamarro, A. (2017). Negative consequences from heavy social networking in adolescents: The mediating role of fear of missing out. Journal of Adolescence, 55, 51-60. doi:10.1016/j. adolescence.2016.12.008

Pempek, T. A., Yermolayeva, Y. A., \& Calvert, S. L. (2009). College students' social networking experiences on Facebook. Journal of Applied Developmental Psychology, 30(3), 227-238. doi:10.1016/j.appdev.2008.12.010

Penninx, B. W., Deeg, D. J., van Eijk, J. T., Beekman, A. T., \& Guralnik, J. M. (2000). Changes in depression and physical decline in older adults: A longitudinal perspective. Journal of Affective Disorders, 61(1-2), 1-12. doi:10.1016/s0165-0327 (00)00152-x

Pontes, H. M., Szabo, A., \& Griffiths, M. D. (2015). The impact of Internet-based specific activities on the perceptions of Internet addiction, quality of life, and excessive usage: A crosssectional study. Addictive Behaviors Reports, 1, 19-25. doi:10.1016/j.abrep.2015.03.002
Radloff, L. S. (1977). The CES-D scale: A self-report depression scale for research in the general population. Applied Psychological Measurement, 1(3), 385-401. doi:10.1177/01466216 7700100306

Rushton, J. L., Forcier, M., \& Schectman, R. M. (2002). Epidemiology of depressive symptoms in the national longitudinal study of adolescent health. Journal of the American Academy of Child \& Adolescent Psychiatry, 41(2), 199-205. doi:10. 1097/00004583-200202000-00014

Selfhout, M. H. W., Branje, S. J. T., Delsing, M., Ter Bogt, T. F. M., \& Meeus, W. H. J. (2009). Different types of Internet use, depression, and social anxiety: The role of perceived friendship quality. Journal of Adolescence, 32(4), 819-833. doi:10.1016/j.adolescence.2008.10.011

Steinfield, C., Ellison, N. B., \& Lampe, C. (2008). Social capital, self-esteem, and use of online social network sites: A longitudinal analysis. Journal of Applied Developmental Psychology, 29(6), 434-445. doi:10.1016/j.appdev.2008.07.002

Stockings, E., Degenhardt, L., Lee, Y. Y., Mihalopoulos, C., Liu, A., Hobbs, M., \& Patton, G. (2015). Symptom screening scales for detecting major depressive disorder in children and adolescents: A systematic review and meta-analysis of reliability, validity and diagnostic utility. Journal of Affective Disorders, 174, 447-463. doi:10.1016/j.jad.2014.11.061

Tang, C. S., \& Koh, Y. Y. (2017). Online social networking addiction among college students in Singapore: Comorbidity with behavioral addiction and affective disorder. Asian Journal of Psychiatry, 25, 175-178. doi:10.1016/j.ajp.2016.10.027

Thapar, A., Collishaw, S., Potter, R., \& Thapar, A. K. (2010). Managing and preventing depression in adolescents. BMJ, 340, c209. doi:10.1136/bmj.c209

Van Gool, C. H., Kempen, G. I. J. M., Penninx, B. W. J. H., Deeg, D. J. H., Beekman, A. T. F., \& Van Eijk, J. T. M. (2003). Relationship between changes in depressive symptoms and unhealthy lifestyles in late middle aged and older persons: Results from the Longitudinal Aging Study Amsterdam. Age and Ageing, 32(1), 81-87. doi:10.1093/ageing/32.1.81

Verkuijl, N. E., Richter, L., Norris, S. A., Stein, A., Avan, B., \& Ramchandani, P. G. (2014). Postnatal depressive symptoms and child psychological development at 10 years: A prospective study of longitudinal data from the South African Birth to Twenty cohort. Lancet Psychiatry, 1(6), 454-460. doi:10.1016/S2215-0366(14)70361-X

Wang, J. C., Xie, H. Y., \& Fisher, J. H. (2009). Multilevel models for discrete outcome meausres. In L.-P. Wang (Ed.), Multilevel models: Applications using $S A S^{(}$(pp. 113-174). Beijing, China: Higher Education Press.

Wang, M., Armour, C., Wu, Y., Ren, F., Zhu, X., \& Yao, S. (2013). Factor structure of the CES-D and measurement invariance across gender in mainland Chinese adolescents. Journal of Clinical Psychology, 69(9), 966-979. doi:10.1002/ jclp. 21978

Wu, A. M. S., Cheung, V. I., Ku, L., \& Hung, E. P. W. (2013). Psychological risk factors of addiction to social networking sites among Chinese smartphone users. Journal of Behavioral Addictions, 2(3), 160-166. doi:10.1556/JBA.2. 2013.006

Yoo, Y.-S., Cho, O.-H., \& Cha, K.-S. (2014). Associations between overuse of the Internet and mental health in adolescents. 
Nursing \& Health Sciences, 16(2), 193-200. doi:10.1111/ nhs. 12086

Young, K. S., \& Rogers, R. C. (1998). The relationship between depression and Internet addiction. CyberPsychology \& Behavior, 1(1), 25-28. doi:10.1089/cpb.1998.1.25
Zhou, S. X., \& Leung, L. (2010). Gratifications, loneliness, leisure boredom and self-esteem as predictors of SNS-game addiction and usage pattern among Chinese college students. Master of Science in New Media, The Chinese University of Hong Kong, Hong Kong. 